\title{
Cellulose Nanofibers Coated with Silver Nanoparticles as a SERS Platform for Detection of Pesticides in Apples
}

Polly Liou ${ }^{1}$, Francois Xavier Nayigiziki ${ }^{1}$, Fanbin Kong ${ }^{2}$, Azlin Mustapha ${ }^{1}$, and Mengshi $\operatorname{Lin}^{1 *}$ 


\section{Abstract}

12 A nanocomposite based on cellulose nanofibers (CNFs) coated with silver nanoparticles (AgNPs)

13 was developed in this study as a flexible and effective substrate for use in surface-enhanced

14 Raman spectroscopy (SERS) analysis. An effective Raman indicator molecule, 4-

15 aminothiophenol (pATP), was used to characterize AgNPs impregnated on CNFs. The CNF-

16 AgNP films were used in SERS analysis to detect thiabendazole (TBZ) pesticides in apples. The

17 influence of $\mathrm{pH}$ on the SERS spectra of TBZ was investigated because TBZ is a neutral molecule

18 that has a low affinity for AgNPs. The pH of TBZ solution was decreased to below the TBZ's

$19 \mathrm{pK}_{\mathrm{a}}$, thus enabling the electrostatic attraction between TBZ and AgNPs. CNFs can prevent the

20 uncontrolled aggregation of AgNPs in low $\mathrm{pH}$ environment and serve as an effective

$21 \mathrm{AgNP} /$ nanocellulose platform for SERS analysis. Results of this study demonstrate that CNF-

22 AgNP nanocomposites can be used to rapidly detect TBZ pesticides in various food products.

23

24 Keywords: SERS; cellulose nanofiber; thiabendazole; pesticides; silver nanoparticles 


\section{Introduction}

26 Nanocellulose has gained much attention recently for its potential applications in material

27 science, biomedicine, agriculture, and food packaging. Nanocellulose is a natural and unique

28 material composed of nanosized cellulose fibrils with a high aspect ratio (length to width) and

29 can self-assemble into well-defined architectures at the nano- or macro-scale. Nanocellulose is

30 one of the most abundant and sustainable nanobiomaterials in nature (Habibi, Lucia, \& Rojas,

31 2010; Hubbe, Rojas, Lucia, \& Sain, 2008; Moon, Martini, Nairn, Simonsen, \& Youngblood,

32 2011). In particular, cellulose nanofibers (CNFs) are made from wood-derived fibers, hence they

33 have a low environmental impact for their production and disposal (Seo et al., 2015).

35 Surface-enhanced Raman Spectroscopy (SERS) is a novel and sensitive technique that can 36 enhance Raman signals of analyte molecules deposited on a roughened metal surface or 37 nanostructure (Zhong Zhang, Yu, Li, Mustapha, \& Lin, 2015; Z. Zhang, Zhang, \& Lin, 2014).

38 SERS can acquire distinctive spectral features based on different vibrational modes of analyte 39 molecules (Shi, Wang, \& Zhan, 2016). The enhancement of SERS signals is based on two 40 mechanisms: electromagnetic enhancement and chemical enhancement. Electromagnetic 41 enhancement is dependent on the excitation of localized surface plasmon on a metal substrate 42 surface with a roughness feature. On the other hand, chemical enhancement is due to the charge 43 transfer between substrate and analyte (Haynes, McFarland, \& Duyne, 2005). The commonly 44 used SERS substrates include gold nanosubstrates, acid-etching silver foils, laser-ablated silver 45 plates, and other conventional SERS substrates based on silicon, glass, and porous alumina 46 (Marques, Nogueira, Pinto, Neto, \& Trindade, 2008). However, these SERS substrates are rigid, 47 brittle, expensive, and not environmentally friendly. Therefore, there remains a need to develop 48 novel, flexible, cost-effective, and biocompatible substrates for SERS analyses. 
50 The objective of this study is to develop a nanocomposite using CNFs impregnated with silver

51 nanoparticles (AgNPs). These CNF-AgNP nanocomposites are lightweight and strong, and can

52 be used as an effective SERS platform (Group, 2013). In addition, CNFs have a large surface

53 area and 3D nanofiber network (Gardner, Oporto, Mills, \& Samir, 2008; Li, Yao, Wang, Cai, \&

54 Wang, 2014), which can increase the potential number of molecules that can react with AgNPs

55 and also enhance the roughness of the substrate's surface to increase SERS signal intensity. An

56 effective Raman indicator molecule, 4-aminothiophenol (pATP), was used to characterize

57 AgNPs on CNFs (Meng, Lai, Jiang, Zhao, \& Zhan, 2013). The performance of CNF-AgNP

58 nanocomposites was evaluated by measuring thiabendazole (TBZ) in different concentrations.

60 TBZ is a pesticide and fungicide that has been widely used post-harvest on fruits and vegetables

61 (USEPA, 2002). Apple is one of the produce that can be contaminated with TBZ (USDA, 2014).

62 Although TBZ has low acute toxicity compared to other pesticides, it has been classified as

63 likely to be carcinogenic at doses high enough to cause disturbance of the thyroid hormone

64 balance (USEPA, 2002). However, TBZ is a hydrophobic molecule that has a low affinity for

65 AgNPs (Alvarez-Puebla \& Liz-Marzan, 2012; Guerrini, Garcia-Ramos, Domingo, \& Sanchez-

66 Cortes, 2009; Nergiz et al., 2013; Stella et al., 2000). This issue can be overcome by controlling

67 the electrostatic force, either altering the surface coating of nanoparticles or changing the

68 solution $\mathrm{pH}$ to enhance the affinity of target molecules and nanoparticle surface (Alvarez-Puebla,

69 Arceo, Goulet, Garrido, \& Aroca, 2005). In this study, we chose the method of adjusting the

70 solution $\mathrm{pH}$ to below TBZ's $\mathrm{pK}_{\mathrm{a}}(\mathrm{pH}=4.65)$ to enhance the SERS signals. To our knowledge,

71 this is the first study to use SERS coupled with CNF-AgNP films for detecting TBZ pesticides in

72 apples. 


\section{2. Materials \& Methods:}

\section{$75 \quad 2.1$ Materials and chemicals}

76 4-aminothiophenol (pATP), ethanol (95\%), $\mathrm{AgNO}_{3}$, thiabendazole (TBZ), 99\% hydrolyzed

77 polyvinyl alcohol (PVA), glycerol, sodium borohydride $\left(\mathrm{NaBH}_{4}\right)$ were all purchased from

78 Sigma-Aldrich (St. Louis, MO). Acetone was purchased from Fisher Scientific (Pittsburgh, PA,

79 USA). Cellulose nanofibers (CNFs) were purchased from the University of Maine.

80

\section{$81 \quad 2.2$ Synthesis of CNF films}

82 Five grams of PVA were dissolved in $50 \mathrm{~g}$ water (10\%) by autoclaving for $30 \mathrm{~min}$. At the same

83 time in another glass flask, $5 \mathrm{~g}$ CNFs were dissolved in $50 \mathrm{~g}$ water $(10 \%), 5 \mathrm{~g}$ of glycerol were

84 added and mixed for $30 \mathrm{~min}$. Then, the two solutions were mixed, heated and stirred at $90^{\circ} \mathrm{C}$ for

$852 \mathrm{~h}$. Lastly, the solution was poured into square plastic petri plates and stored at room

86 temperature for $24 \mathrm{~h}$ to form a film.

87

$88 \quad 2.3$ Impregnation of AgNPs into CNF films

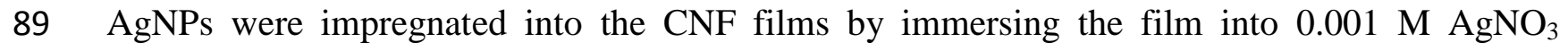

90 solution for $1 \mathrm{~h}$ at room temperature. CNF films were then rinsed by ethanol to wash out the

91 chemicals. The silver ion-saturated CNF films were placed in $0.001,0.01$, and $0.1 \mathrm{M} \mathrm{NaBH}_{4}$

92 solution for $10 \mathrm{~min}$ in an ice bath, and the color of the films gradually changed from bright

93 yellow to brown yellow as the molar ratio of $\mathrm{NaBH}_{4}$ increased. The CNF-AgNP nanocomposites

94 were rinsed with Milli-Q water to remove the excessive chemicals on the films.

95

$96 \quad 2.4$ Preparation of pATP solution 
97 pATP powders were mixed with $95 \%$ ethanol to make a series of concentrations of pATP

98 solutions (1, 5, and $10 \mathrm{ppm})$. A small amount of pATP solution was added onto the CNF-AgNP

99 nanocomposites. pATP was used as a Raman reporter to conjugate with AgNPs to enhance

100 Raman signals. The film samples were then measured by SERS to collect the signals of AgNPs-

101 pATP complexes.

102

103

$2.5 \mathrm{UV}$-vis spectroscopy for detection of AgNPs prepared with different concentration of $\mathrm{NaBH}_{4}$

104 A volume of $30 \mathrm{~mL}$ of $0.001,0.01$, and $0.1 \mathrm{M}$ of $\mathrm{NaBH}_{4}$ was prepared in Erlenmeyer flasks. The

105 solutions were then stirred and cooled in an ice bath for $20 \mathrm{~min}$. Then, $0.001 \mathrm{M}$ of $\mathrm{AgNO}_{3}$ was

106 then added at a rate of 1 drop per second into different concentrations of $\mathrm{NaBH}_{4}$ solution. The

107 solution was stirred continually until the last drop of $\mathrm{AgNO}_{3}$ was added. This step was carefully

108 controlled because if stirring was continued after all the $\mathrm{AgNO}_{3}$ was added, the AgNPs would

109 aggregate and the color of the solution would become gray. Finally, the three yellow AgNP

110 solutions were obtained using different molar ratios of $\mathrm{NaBH}_{4}: \mathrm{AgNO}_{3}$ and the solutions were

111 tested by UV-vis spectroscopy. CNF-AgNP nanocomposites were then used as a SERS substrate

112 to test the performance of this substrate. The pATP molecules were chosen as the target analyte

113 in the measurement.

114

1152.6 Preparation of different concentrations of TBZ pesticide at different $p H$ values

116 TBZ was another analyte for SERS analysis. In this study, we synthesized 1, 5, 10, and $100 \mathrm{ppm}$

117 of TBZ by dissolving TBZ powders in acetone solution. Each sample was then measured at

118 different $\mathrm{pH}$ values $\left(4,6\right.$, and 7). The $\mathrm{pH} 4$ is below TBZ's $\mathrm{pK}_{\mathrm{a}}$ value of 4.65 . Then, a volume of

$119200 \mu \mathrm{L}$ of different concentrations and different $\mathrm{pH}$ values of TBZ solutions were added into 2 $120 \mathrm{~cm}^{2}$ CNF-AgNPs film for $1 \mathrm{~min}$ before SERS detection. 


\section{2.7 Detection of TBZ in apple}

123 Apple samples were cut into small pieces and spiked with different concentrations of TBZ. TBZ

124 was extracted from the apple by mixing the spiked apple $(0.5 \mathrm{~g})$ with $50 \%$ of acetonitrile-water 125 solution $(0.5 \mathrm{~mL})$. Then, the mixture was shaken for $10 \mathrm{~min}$ and centrifuged at $3354 \mathrm{G}$-force for $12610 \mathrm{~min}$. A drop of supernatant was then placed on the CNF-AgNPs substrate for SERS analysis.

128 2.8 SERS measurement to detect pATP and TBZ

129 Droplets of TBZ samples were deposited on the CNF-AgNP substrate before SERS measurement.

130 SERS was conducted by a Raman spectrometer (Renishaw 1000, Renishaw Inc., Gloucestershire, 131 UK) equipped with a $785 \mathrm{~nm}$ laser and $50 \times$ objective. The laser beam $(25 \mathrm{~mW})$ was focused 132 directly on the gold slide. OMNIC software was used to collect the Raman signals from ten 133 randomly selected spots for each droplet.

136 Delight software (D-Squared Development Inc., LaGrande, OR, USA) was used to analyze the

137 SERS spectra from different treatments. First, the collected spectra from 10 different locations of 138 different samples were averaged to a final spectrum. Second, the spectra were smoothed to 139 reduce the noises. The SERS spectra of pATP were acquired in the wavenumber range between 140300 to $1800 \mathrm{~cm}^{-1}$, and the SERS spectra of TBZ were acquired in the wavenumber range 141 between 700 to $1600 \mathrm{~cm}^{-1}$.

\section{3. Results \& Discussions}

1443.1 Characterization of AgNPs in CNFs by UV-vis spectroscopy and TEM 
145 UV-vis spectroscopy was used to characterize AgNPs generated in the mixtures of $0.001 \mathrm{M}$ $146 \mathrm{AgNO}_{3}$ solution with $0.001,0.01$, and $0.1 \mathrm{M}$ reducing agent, $\mathrm{NaBH}_{4}$ solution. Due to the surface 147 plasmon resonance (SPR) of conducting electron on the surface of AgNPs, the synthesized 148 AgNPs display one absorption feature as shown in Figure 1. This is in accord with previous 149 studies that AgNPs showed a characteristic optical absorption peak at 400 nm (Bhui et al., 2009;

150 Martinez-Castanon, Nino-Martinez, Martinez-Gutierrez, Martinez-Mendoza, \& Ruiz, 2008).

(a)

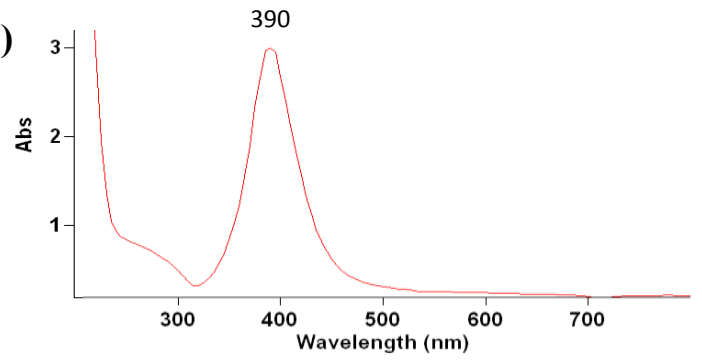

(b)

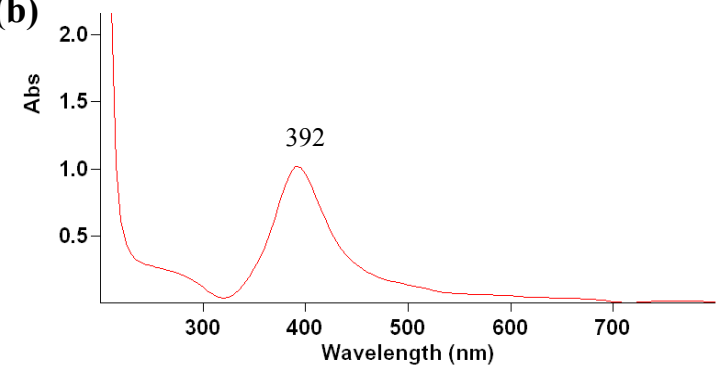

(c)

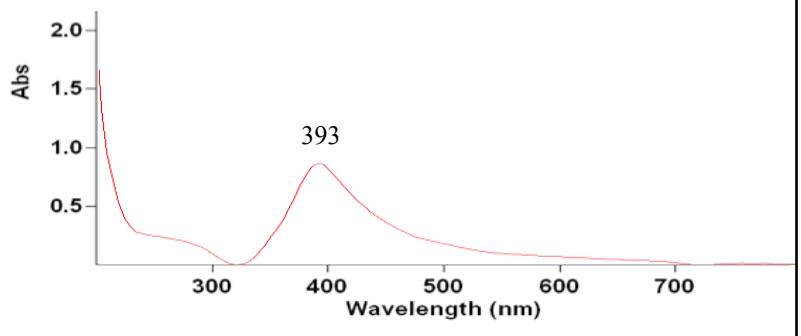

152 Figure 1. Absorption spectra of silver nanoparticles prepared with different $\mathrm{NaBH}_{4}: \mathrm{AgNO}_{3}$ 153 molar ratios: 100:1 (a); 10:1 (b); 1:1 (c).

155 In Figure 1, the $\mathrm{NaBH}_{4}: \mathrm{AgNO}_{3}$ molar ratio of 100:1 showed a narrow peak at $390 \mathrm{~nm}$. However, 156 when the concentration of $\mathrm{NaBH}_{4}$ decreased, the absorption band became broader. The peak was 157 located at $392 \mathrm{~nm}$ when the $\mathrm{NaBH}_{4}: \mathrm{AgNO}_{3}$ molar ratio was 10:1; and the sample with $158 \mathrm{NaBH}_{4}: \mathrm{AgNO}_{3}$ molar ratio of 1:1 even exhibited a much broadened peak at $393 \mathrm{~nm}$. The red159 shift and the bandwidth of the absorption spectra indicated the increased size of AgNPs and a 160 decrease in the reaction efficiency as the concentration of $\mathrm{NaBH}_{4}$ is reduced. Therefore, it is 161 speculated that the $\mathrm{NaBH}_{4}: \mathrm{AgNO}_{3}$ molar ratio of 1:1 formed the largest sized AgNPs among the 
162 three molar ratios. The possible reason is that there was a small amount of reducing agent,

$163 \mathrm{NaBH}_{4}$, to reduce the ionic silver, so the excessive silver atoms aggregated together. In contrast,

164 the $\mathrm{NaBH}_{4}: \mathrm{AgNO}_{3}$ molar ratio of 100:1 formed the smallest size of $\mathrm{AgNPs}$, which was due to

165 more free electron generated from $\mathrm{NaBH}_{4}$ to prevent the aggregation of silver (Maneerung,

166 Tokura, \& Rujiravanit, 2008).
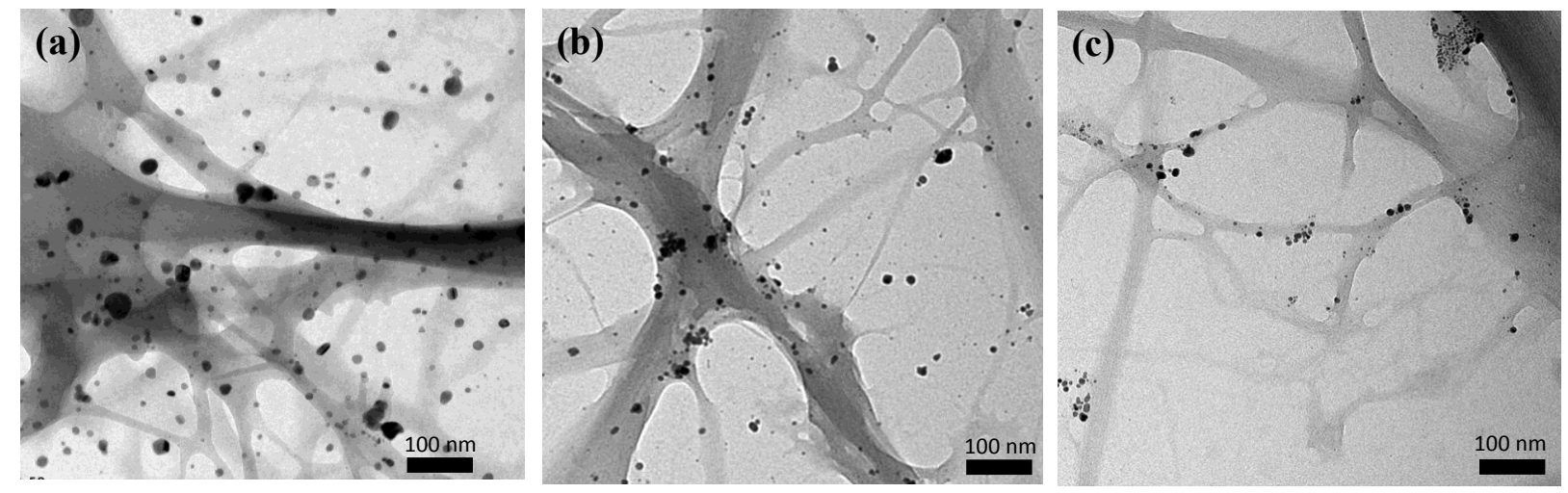

168 Figure 2. TEM images of AgNPs impregnated CNFs prepared with different $\mathrm{NaBH}_{4}: \mathrm{AgNO}_{3}$ molar ratios: $1: 1$ with average size: $20.05 \pm 8.28$ (a); 10:1with average size: $9.44 \pm 4.73$ (b); 100:1 with average size: $4.71 \pm 4.40$ (c).

172 TEM images were obtained in this study for the CNFs impregnated with AgNPs that were

173 prepared by three different $\mathrm{NaBH}_{4}: \mathrm{AgNO}_{3}$ molar ratios (Figure 2). The images show 3D

174 nanofiber network and nano-sized structure of cellulose fibers with high length to width ratio and

175 a large surface area that can load AgNPs. Moderate amounts of AgNPs are loaded and they are

176 evenly distributed on the surface of CNFs. The average size of AgNPs made with the

$177 \mathrm{NaBH}_{4}: \mathrm{AgNO}_{3}$ molar ratio of $1: 1$ was estimated to be $20.05 \mathrm{~nm}$ (Figure 2a). When the

$178 \mathrm{NaBH}_{4}: \mathrm{AgNO}_{3}$ molar ratio increased to 10:1, the average size of AgNPs became smaller at 9.44

$179 \mathrm{~nm}$ (Figure 2b). AgNPs made with the $\mathrm{NaBH}_{4}: \mathrm{AgNO}_{3}$ molar ratio of 100:1 had the smallest

180 particle size of $4.71 \mathrm{~nm}$ (Figure 2c). The size distribution of AgNPs on CNF with different

$181 \mathrm{NaBH}_{4}: \mathrm{AgNO}_{3}$ molar ratios is shown in Figure 3. 

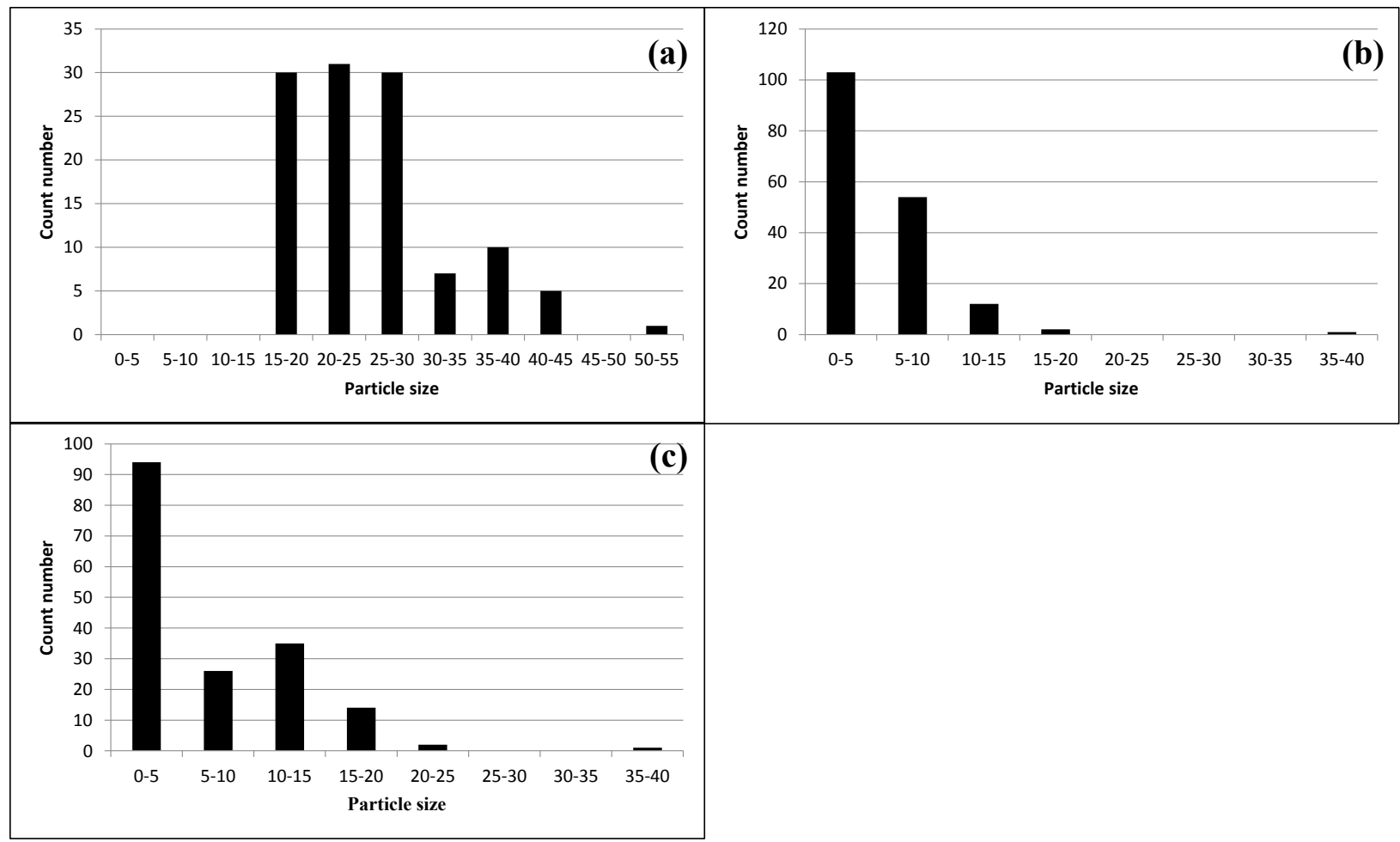

184 Figure 3. The size distribution of $\mathrm{AgNPs}$ on $\mathrm{CNF}$ with $\mathrm{NaBH}_{4}: \mathrm{AgNO}_{3}$ molar ratio of: (a) 1:1; (b)

185 10:1; and (c) 100:1.

187 The size and size distribution of nanoparticles influence the properties and the enhancement of 188 SERS activities (Agnihotri, Mukherji, \& Mukherji, 2014; Israelsen, Hanson, \& Vargis, 2015).

189 The intensities of SERS measurement from AgNPs from high to low was in accord with the $190 \mathrm{NaBH}_{4}: \mathrm{AgNO}_{3}$ molar ratio of 10:1 > 1:1>100:1 (Figure 4). The AgNPs made with the $191 \mathrm{NaBH}_{4}: \mathrm{AgNO}_{3}$ molar ratio of 10:1 had the strongest SERS intensity compared to the other two 192 ratios. This is because different molar ratio of $\mathrm{NaBH}_{4}: \mathrm{AgNO}_{3}$ resulted in the formation of 193 different sizes of AgNPs, which affect the intensity of Raman signals. For the $\mathrm{NaBH}_{4}: \mathrm{AgNO}_{3}$ 194 molar ratio of $1: 1$, the amount of reducing agent $\left(\mathrm{NaBH}_{4}\right)$ is too low. Therefore, AgNPs 195 aggregated together and affected the SERS detection. Due to their small size and low mass, once 196 NPs bind together it is often difficult to separate them. On the other hand, the $\mathrm{NaBH}_{4}: \mathrm{AgNO}_{3}$ 197 molar ratio of 100:1 had the weakest SERS intensity due to the smallest particle size of AgNPs. 
198 Hence, the $\mathrm{NaBH}_{4}: \mathrm{AgNO}_{3}$ molar ratio of 10:1 was chosen to be used as the CNF-AgNPs 199 substrate due to the highest SERS signals among the three types of substrates made with 200 different concentrations of $\mathrm{NaBH}_{4}: \mathrm{AgNO}_{3}$ molar ratio solutions.

201

(a)

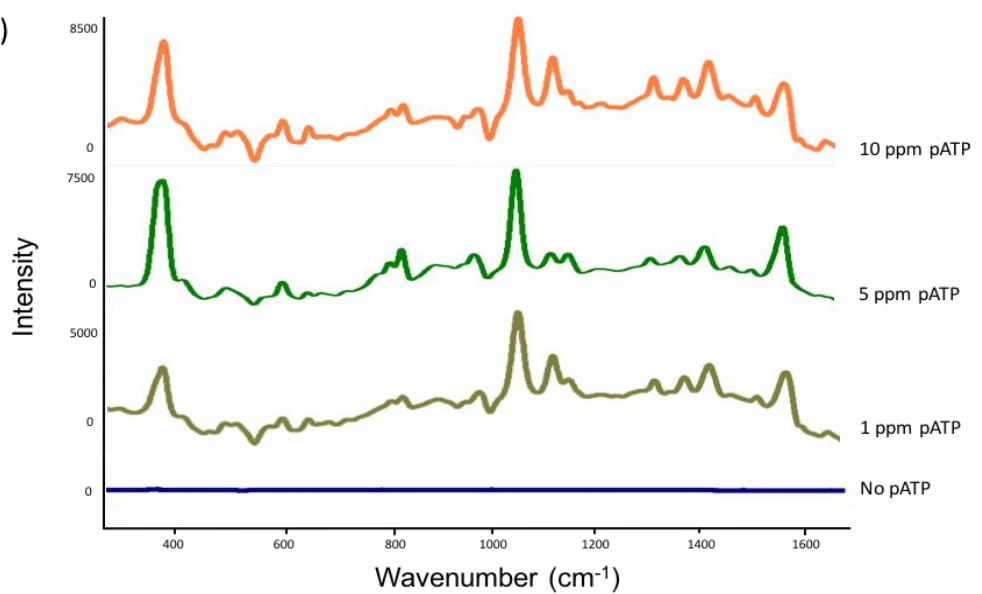

(b)

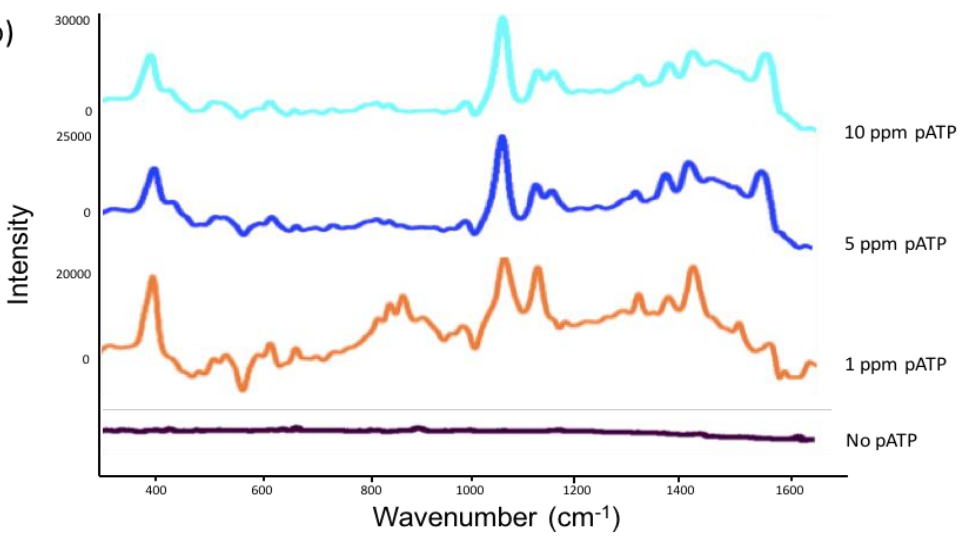

(c)

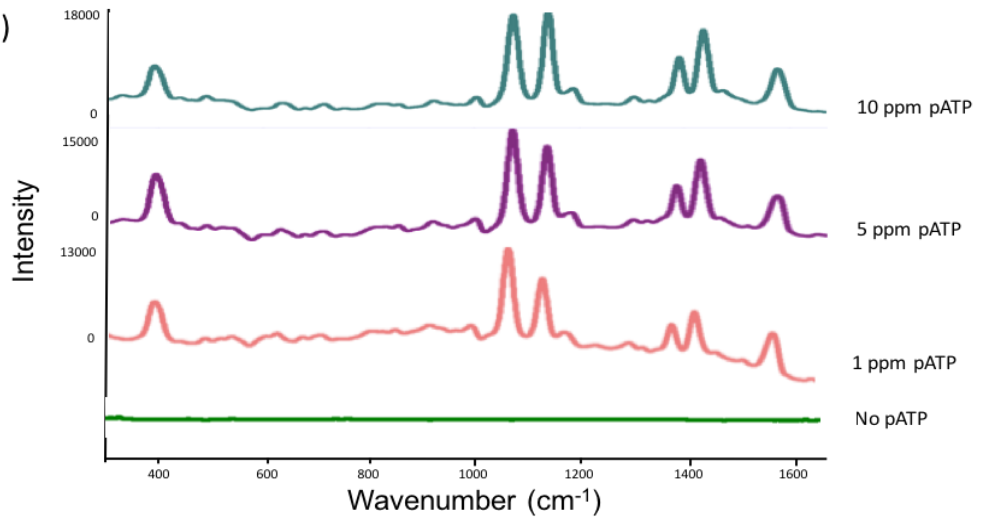

204 Figure 4. SERS spectra of pATP-AgNPs complexes prepared with different $\mathrm{NaBH}_{4}: \mathrm{AgNO}_{3}$ molar ratio of: (a) 1:1; (b) 10:1; and (c) 100:1. 
208 pATP was used in SERS measurement to bind with AgNPs, they can generate the vibrational 209 signals under SERS measurement. Figure 4 shows five significant Raman peaks of pATP-AgNPs 210 complexes at $\sim 390,1078,1143,1435$, and $1590 \mathrm{~cm}^{-1}$. The peak located at $\sim 390 \mathrm{~cm}^{-1}$ is assigned 211 to the bending mode of the C-S bond (Wu et al., 2011; Zhou, Zhi, Zhao, \& Xu, 2010). The bands 212 at $\sim 1078$ and $1590 \mathrm{~cm}^{-1}$ are assigned to the $\mathrm{a}_{1}$ vibrational modes (in-plane modes). A peak at

$213 \sim 1078 \mathrm{~cm}^{-1}$ is attributed the C-S stretching vibration, and C-C stretching vibrations for a peak at

$214 \sim 1590 \mathrm{~cm}^{-1}$. Another vibrational mode of pATP-AgNPs complexes is called $\mathrm{b}_{2}$ modes (in plane 215 mode), which are located at $\sim 1143$ and $1435 \mathrm{~cm}^{-1}$. The $\mathrm{b}_{2}$ vibrational modes are based on the 216 charge transfer condition of SERS, which is the charge transfer between the AgNPs and the 217 adsorbed molecules (Premkumar \& Geckeler, 2014). These peaks are similar to the SERS 218 spectra of pATP-AgNPs complexes measured in our previous studies, so we extrapolated that 219 AgNPs were successfully impregnated onto the CNF films. In addition, different concentrations 220 of pATP and different concentrations of $\mathrm{NaBH}_{4}$ can both affect the intensity of SERS signals

221 (Figure 4). SERS is a highly sensitive method that doesn't require a lot of metallic NPs in the 222 substrate, but can still generate very strong Raman signals. As shown in Figure 2, moderate 223 amounts of AgNPs loaded on the surface of CNFs are sufficient to generate strong SERS singals 224 as shown in Figure 4.

225

2263.3 Detection of TBZ using CNF-AgNPs nanocomposites as SERS substrate

227 Figure 5 shows a Raman spectrum of TBZ powders (99\%) and a SERS spectrum of TBZ 228 solutions measured by CNF-AgNP nanocomposites. The powder form and the solution of TBZ 229 exhibit similar Raman peaks at 785,1280 , and $1580 \mathrm{~cm}^{-1}$. However, SERS spectra of the TBZ 230 solution displayed more visible peaks than the normal Raman spectra of TBZ powders. Figure 5 
231 shows the SERS spectra of different concentrations (1, 10, 50, and $100 \mathrm{ppm})$ of TBZ measured 232 by the CNF-AgNP substrate. Only when the $\mathrm{pH}$ level is below TBZ's $\mathrm{pK}_{\mathrm{a}}$, were strong SERS

233 signals obtained. Because TBZ is a neutral and hydrophobic molecule, it has a low affinity for 234 the negatively charged AgNP surface due to the lack of electrostatic attractions. TBZ molecule 235 contains secondary amine groups, which can be protonated at $\mathrm{pH}$ values below their respective $236 \mathrm{pK}_{\mathrm{a}}$ values (Wei \& Vikesland, 2015). Therefore, 0.1 M HCL was added to reduce the $\mathrm{pH}$ of the 237 TBZ solution to $\sim 4$ to induce electrostatic attraction between the amine groups of TBZ and the 238 borohydride ions $\left(\mathrm{BH}_{4}^{-}\right)$of AgNPs (Figure 7). However, nanoparticles can aggregate when they 239 are in a low $\mathrm{pH}$ environment, which will affect SERS measurement (Alvarez-Puebla \& Aroca, 240 2009). Nanocellulose is acid resistant, chemically stable, and in a 3D rigid scaffold structure

241 (Wei \& Vikesland, 2015). Our results demonstrate that this new SERS substrate is uniform and 242 very stable because AgNPs are highly uniform and firmly adhered to the CNFs. Cellulose 243 nanofibers have unique properties of porous structure and active hydroxyl groups on their 244 surface, which can function as a substrate for AgNPs. The hydroxyl groups can act as electron 245 donors to aid in the formation of AgNPs (Lin \& Tang, 2015). This CNF-AgNP platform can 246 prevent the uncontrolled aggregation of AgNPs in low $\mathrm{pH}$ environments.

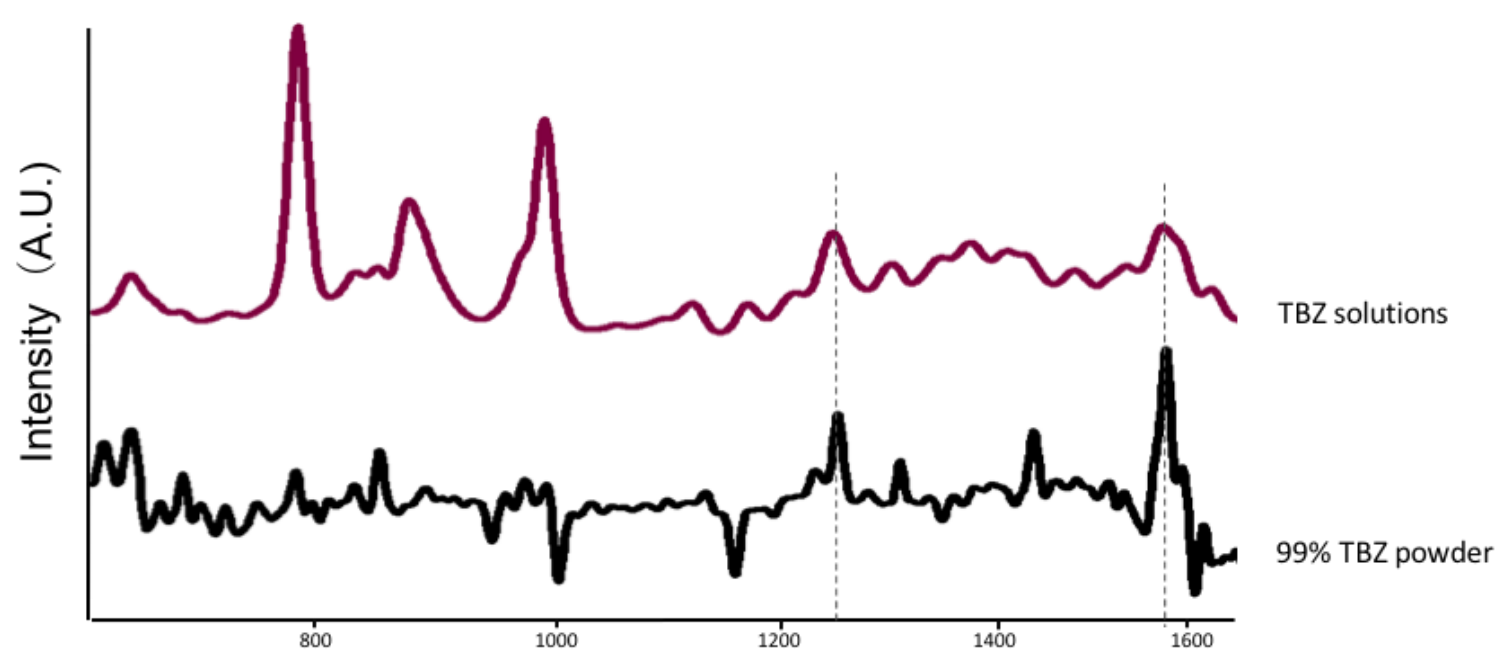

248 Figure 5. A Raman spectrum of TBZ powders and a SERS spectrum of TBZ solutions that were measured using CNF-AgNP films. 
251 Different concentrations of TBZ solutions (1, 10, 50, and $100 \mathrm{ppm})$ were measured by the CNF252 AgNP SERS substrate and the spectra (Figure 6) exhibited four prominent peaks at 785, 1010, 2531280 , and $1580 \mathrm{~cm}^{-1}$. The assignments to these peaks were out of plane bending of C-H, out of 254 plane bending of $\mathrm{C}-\mathrm{C}-\mathrm{C}$, ring stretching, and $\mathrm{C}=\mathrm{N}$ stretching, respectively (Kim, Kim, Lee, Jung, 255 \& Lee, 2009). In addition, TBZ in real food samples (apples) was also detected in this study. 256 TBZ solutions (1, 10, 50, $100 \mathrm{ppm})$ were applied onto apples and then extracted from the apples 257 for SERS analysis. Figure 8 shows that the SERS spectra of TBZ extracted from the apple have 258 similar peaks with that of pure TBZ solutions (Figure 6), but the intensity of SERS signals were 259 not as strong as that of pure TBZ solutions. Moreover, the limit of detection of 5 ppm for TBZ 260 were obtained, which is lower than the current tolerance ppm for apple and other citrus fruits (10 $261 \mathrm{ppm})(\mathrm{EPA}, 2002)$. 
(a)
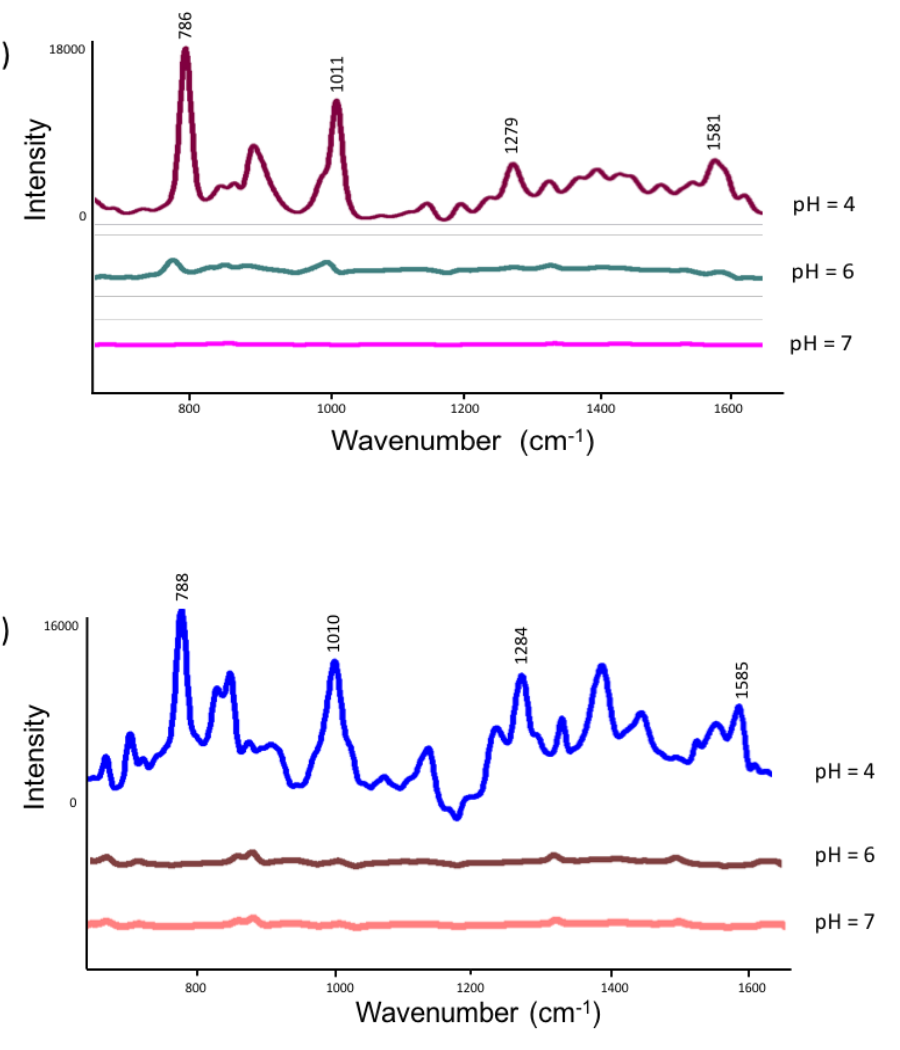

(c)

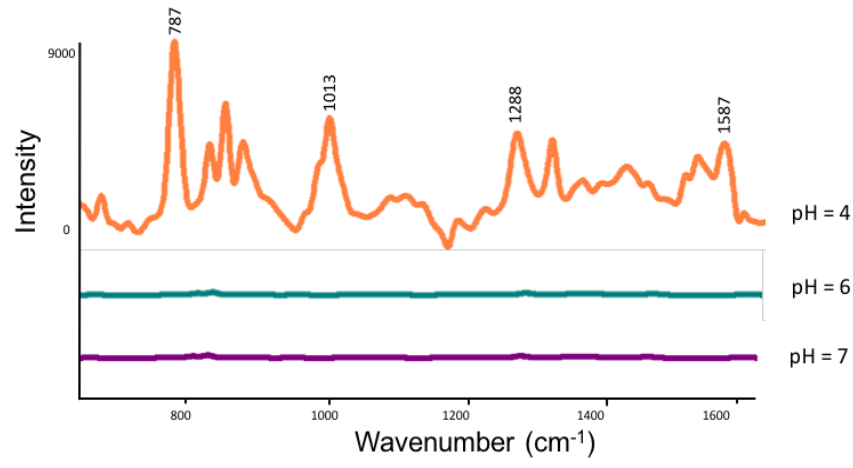

(b)

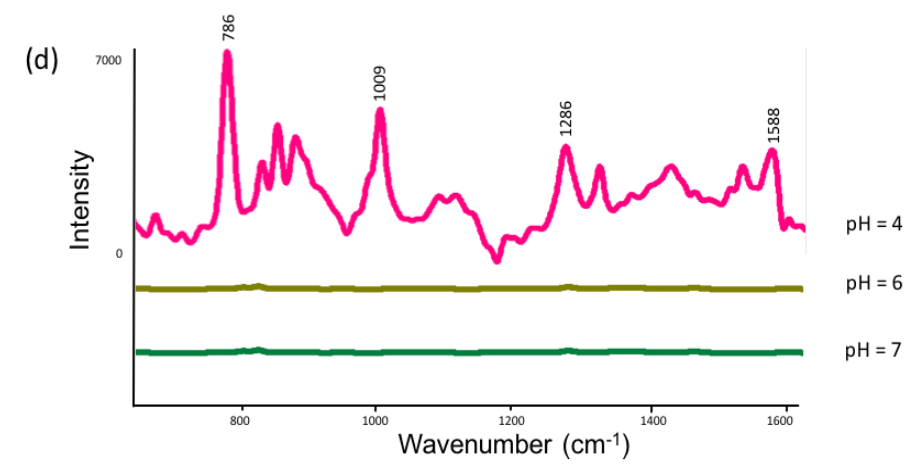

Figure 6. SERS spectra of different concentrations of TBZ measured by CNF-AgNP substrate: (a) 100 ppm TBZ; (b) 50 ppm TBZ; (c) 10 ppm TBZ; (d) 1 ppm TBZ. 


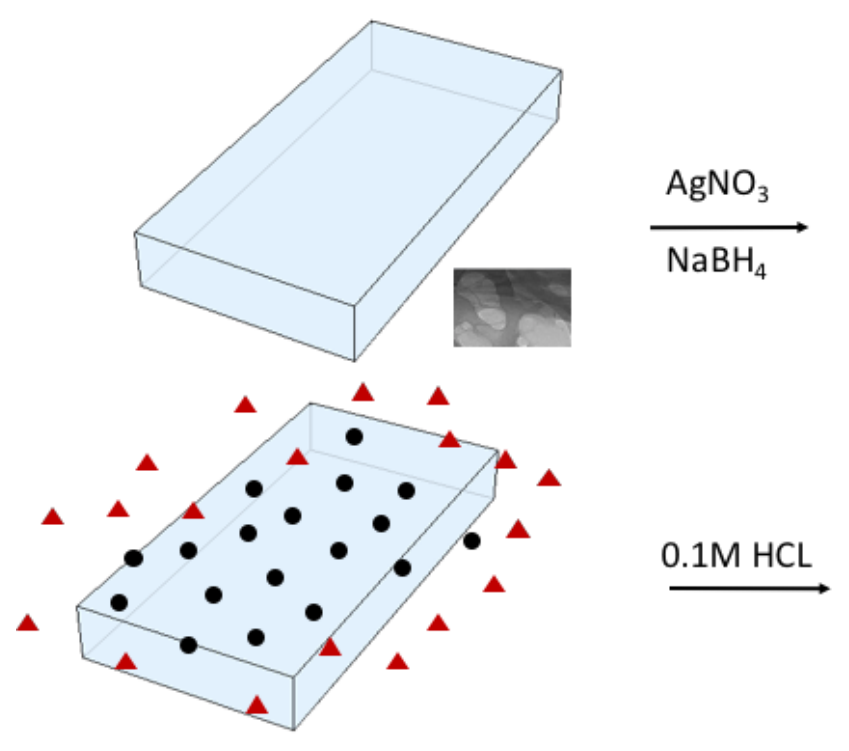

TBZ at environmental $\mathrm{pH}$
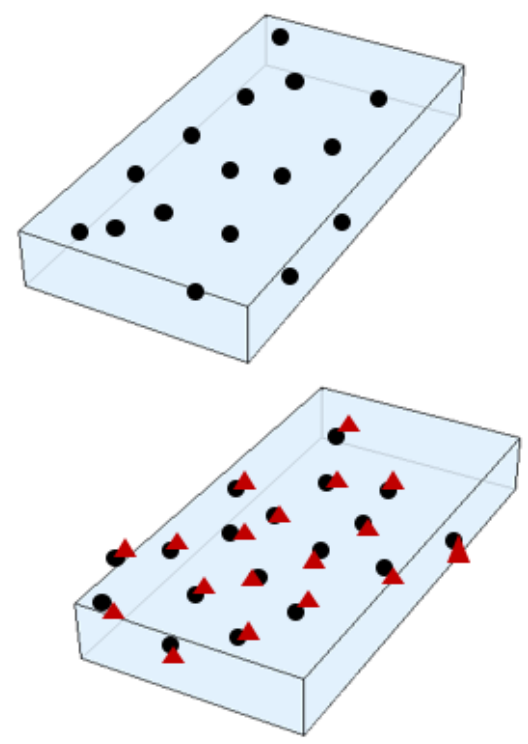

TBZ below pKa pH $(<4.65)$

CNFs

- AgNPs

- TBZ

271 Figure 7. A schematic diagram of $\mathrm{pH}$-induced adsorption of TBZ on CNF-AgNP substrate.

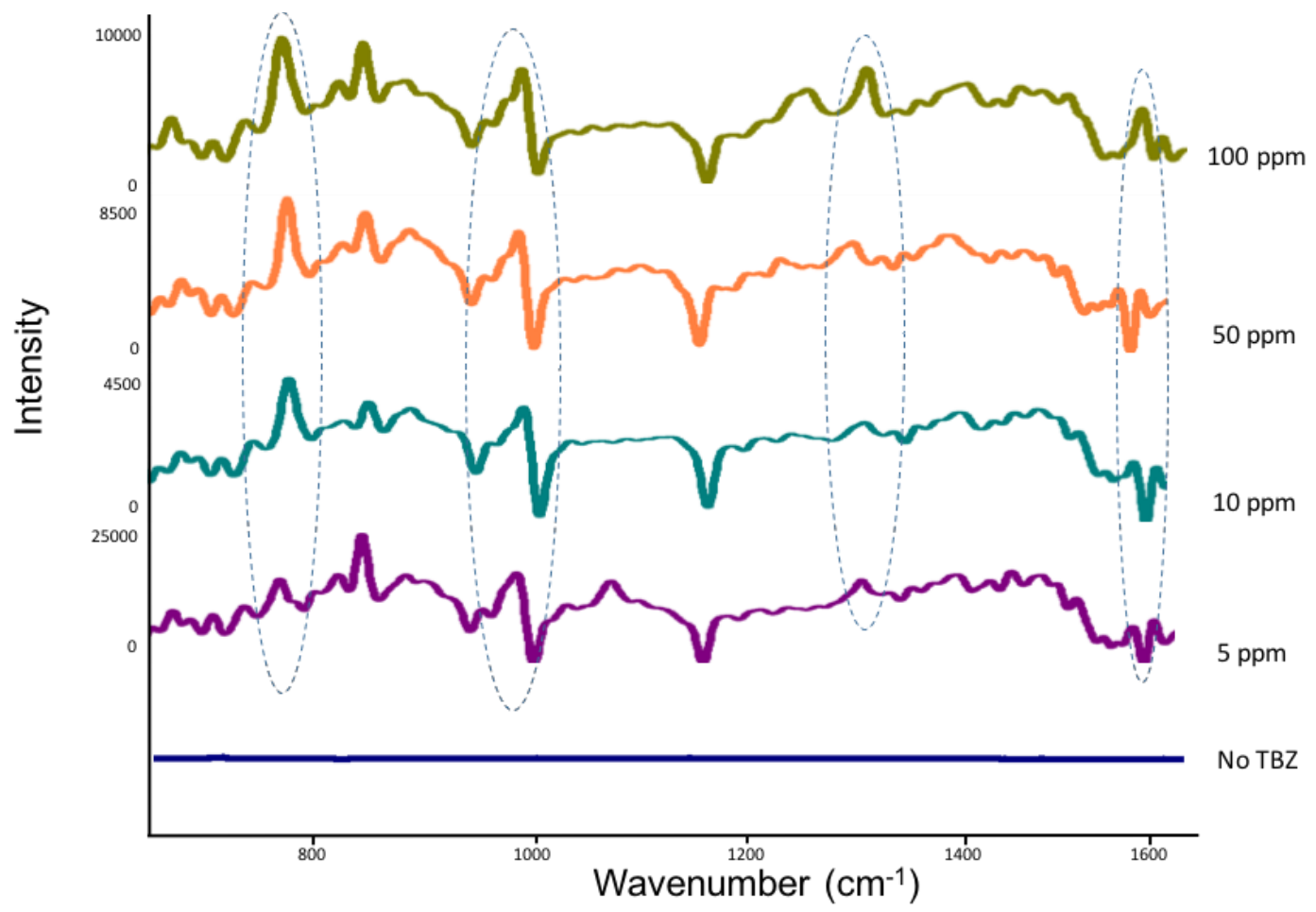

273 Figure 8. SERS spectra of different concentrations of TBZ extracted from apples. 


\section{4. Conclusions}

276 In this study, CNF-AgNP nanocomposite substrates were developed. They are flexible and 277 environmentally friendly compared to the traditional SERS substrates. This novel SERS 278 substrate was evaluated by measuring pATP and TBZ pesticide. The AgNPs-pATP complexes 279 exhibit characteristic SERS peaks at $\sim 390,1078,1143,1435$, and $1590 \mathrm{~cm}^{-1}$. On other hand, the 280 SERS spectra of TBZ show prominent peaks at $\sim 785,1010,1280$, and $1580 \mathrm{~cm}^{-1}$. TBZ is a 281 neutral and hydrophobic molecule that has a low affinity for the surface of negatively charged 282 AgNPs. Therefore, TBZ only exhibited strong SERS signals when the $\mathrm{pH}$ is below the TBZ's $\mathrm{pk}_{\mathrm{a}}$ 283 value $(\mathrm{pH}=4.65)$ based on the electrostatic attractions between AgNPs and TBZ. This method is 284 simple, rapid, sensitive, and can be extended to rapid detection of other neutral molecules and 285 pesticides in various food products.

287 Acknowledgements

288 We acknowledge the assistance from the Electron Microscopy Core in electron microscopy 289 analysis at University of Missouri. This study is supported by USDA NIFA nanotechnology 290 program (2016-67021-24994). 
References:

292

293

294

295

296

297

298

299

300

301

302

303

304

305

306

307

308

309

310

311

312

313

314

315

316

317

318

319

320

321

322

323

324

325

326

327

328

329

330

331

332

Agnihotri, S., Mukherji, S., \& Mukherji, S. (2014). Size-controlled silver nanoparticles synthesized over the range $5-100 \mathrm{~nm}$ using the same protocol and their antibacterial efficacy. RSC Adv., 4(8), 3974-3983.

Alvarez-Puebla, R. A., Arceo, E., Goulet, P. J., Garrido, J. J., \& Aroca, R. F. (2005). Role of nanoparticle surface charge in surface-enhanced Raman scattering. J. Phys. Chem. $B$, 109(9), 3787-3792.

Alvarez-Puebla, R. A., \& Aroca, R. F. (2009). Synthesis of silver nanoparticles with controllable surface charge and their application to surface-enhanced Raman scattering. Anal. Chem., 81(6), 2280-2285.

Alvarez-Puebla, R. A., \& Liz-Marzan, L. M. (2012). Traps and cages for universal SERS detection. Chem. Soc. Rev., 41(1), 43-51.

Bhui, D. K., Bar, H., Sarkar, P., Sahoo, G. P., De, S. P., \& Misra, A. (2009). Synthesis and UV-vis spectroscopic study of silver nanoparticles in aqueous SDS solution. J. Mol. Liq., 145(1), 33-37.

EPA. Reregistration Eligibility Decision (RED) Thiabendazole (2002). file:///Users/pollyliou/Downloads/red_PC-060101_1-May-02.pdf Accessed May, 30, 2016.

Gardner, D. J., Oporto, G. S., Mills, R., \& Samir, M. A. S. A. (2008). Adhesion and surface issues in cellulose and nanocellulose. J. Adhes. Sci. Technol., 22(5-6), 545-567.

Group, N. P. Cellulose nanofiber manufacturing technology and application development. (2013). http://www.nipponpapergroup.com/english/research/organize/cnf.html Accessed May 30, 2016.

Guerrini, L., Garcia-Ramos, J. V., Domingo, C., \& Sanchez-Cortes, S. (2009). Nanosensors based on viologen functionalized silver nanoparticles: few molecules surface-enhanced Raman spectroscopy detection of polycyclic aromatic hydrocarbons in interparticle hot spots. Anal. Chem., 81(4), 1418-1425.

Habibi, Y., Lucia, L. A., \& Rojas, O. J. (2010). Cellulose nanocrystals: chemistry, self-assembly, and applications. Chem. Rev., 110(6), 3479-3500.

Haynes, C. L., McFarland, A. D., \& Duyne, R. P. V. (2005). Surface-enhanced Raman spectroscopy. Anal. Chem., 77(17), 338 A-346 A.

Hubbe, M. A., Rojas, O. J., Lucia, L. A., \& Sain, M. (2008). Cellulosic nanocomposites: a review. Bioresourses, 3(3), 929-980.

Israelsen, N. D., Hanson, C., \& Vargis, E. (2015). Nanoparticle properties and synthesis effects on surface-enhanced Raman scattering enhancement factor: an introduction. Scientific World J., 2015.

Kim, M.-S., Kim, M.-K., Lee, C.-J., Jung, Y.-M., \& Lee, M.-S. (2009). Surface-enhanced Raman spectroscopy of benzimidazolic fungicides: benzimidazole and thiabendazole. Bull. Korean Chem. Soc., 30(12), 2930-2934.

Li, Z., Yao, C., Wang, F., Cai, Z., \& Wang, X. (2014). Cellulose nanofiber-templated threedimension TiO2 hierarchical nanowire network for photoelectrochemical photoanode. Nanotechnology, 25(50), 504005. 
Lin, Y.-W., \& Tang, C. (2015). Electrochemical synthesis and deposition of surface-enhanced Raman scattering-active silver microstructures on a screen-printed carbon electrode. The Journal of Physical Chemistry C, 119(44), 24865-24874.

Maneerung, T., Tokura, S., \& Rujiravanit, R. (2008). Impregnation of silver nanoparticles into bacterial cellulose for antimicrobial wound dressing. Carbohydr. Polym., 72(1), 43-51.

Marques, P. A., Nogueira, H. I., Pinto, R. J., Neto, C. P., \& Trindade, T. (2008). Silver-bacterial cellulosic sponges as active SERS substrates. J. Raman Spectrosc., 39(4), 439-443.

Martinez-Castanon, G., Nino-Martinez, N., Martinez-Gutierrez, F., Martinez-Mendoza, J., \& Ruiz, F. (2008). Synthesis and antibacterial activity of silver nanoparticles with different sizes. J. Nanopart. Res., 10(8), 1343-1348.

Meng, Y., Lai, Y., Jiang, X., Zhao, Q., \& Zhan, J. (2013). Silver nanoparticles decorated filter paper via self-sacrificing reduction for membrane extraction surface-enhanced Raman spectroscopy detection. Analyst, 138(7), 2090-2095.

Moon, R. J., Martini, A., Nairn, J., Simonsen, J., \& Youngblood, J. (2011). Cellulose nanomaterials review: structure, properties and nanocomposites. Chem. Soc. Rev., 40(7), 3941-3994.

Nergiz, S. Z., Gandra, N., Farrell, M. E., Tian, L., Pellegrino, P. M., \& Singamaneni, S. (2013). Biomimetic SERS substrate: peptide recognition elements for highly selective chemical detection in chemically complex media. J. Mater. Chem. A, 1(22), 6543-6549.

Premkumar, T., \& Geckeler, K. E. (2014). Facile synthesis of silver nanoparticles using unmodified cyclodextrin and their surface-enhanced Raman scattering activity. New J. Chem., 38(7), 2847-2855.

Seo, J.-H., Chang, T.-H., Lee, J., Sabo, R., Zhou, W., Cai, Z., . . Ma, Z. (2015). Microwave flexible transistors on cellulose nanofibrillated fiber substrates. Appl. Phys. Lett., 106(26), 262101.

Shi, Y.-e., Wang, W., \& Zhan, J. (2016). A positively charged silver nanowire membrane for rapid on-site swabbing extraction and detection of trace inorganic explosives using a portable Raman spectrometer. Nano Research, 9(8), 2487-2497.

Stella, B., Arpicco, S., Peracchia, M. T., Desmaële, D., Hoebeke, J., Renoir, M., . . Couvreur, P. (2000). Design of folic acid-conjugated nanoparticles for drug targeting. J. Pharm. Sci., 89(11), 1452-1464.

USDA. USDA pestcide data program.(2014). https://www.ams.usda.gov/sites/default/files/media/2014 PDP Annual Summary.pdf Accessed May 18, 2016.

USEPA. EPA reregistration eligibility decision thabendazole.(2002). https://www3.epa.gov/pesticides/chem search/reg actions/reregistration/fs PC060101 1-May-02.pdf Accessed June 1, 2015.

Wei, H., \& Vikesland, P. J. (2015). pH-triggered molecular alignment for reproducible SERS detection via an AuNP/nanocellulose platform. Sci. Rep., 5, 18131.

Wu, D.-Y., Zhao, L.-B., Liu, X.-M., Huang, R., Huang, Y.-F., Ren, B., \& Tian, Z.-Q. (2011). Photondriven charge transfer and photocatalysis of $p$-aminothiophenol in metal nanogaps: a DFT study of SERS. Chem. Comm., 47(9), 2520-2522.

Zhang, Z., Yu, Q., Li, H., Mustapha, A., \& Lin, M. (2015). Standing gold nanorod arrays as reproducible SERS substrates for measurement of pesticides in apple juice and vegetables. J. Food Sci., 80(2), N450-N458. 
377 Zhang, Z., Zhang, S., \& Lin, M. (2014). DNA-embedded Au-Ag core-shell nanoparticles

378 assembled on silicon slides as a reliable SERS substrate. Analyst, 139(9), 2207-2213.

379 Zhou, Y., Zhi, J., Zhao, J., \& Xu, M. (2010). Surface-Enhanced Raman Scattering of 4-

380 Aminothiophenol Adsorbed on Silver Nanosheets Deposited onto Cubic Boron Nitride

381

382

Films. Anal. Sci., 26(9), 957-961. 